\title{
Nail-Patella syndrome with early onset end-stage renal disease in a child with a novel heterozygous missense mutation in the LMX1B homeodomain: A case report
}

\author{
SOLEDAD CARINELLI ${ }^{1}$, OLALLA ALVAREZ BLANCO ${ }^{2}$, \\ ANA PERDOMO-RAMIREZ ${ }^{1}$ and FELIX CLAVERIE-MARTIN ${ }^{1}$ \\ ${ }^{1}$ Unidad de Investigación, Hospital Universitario Nuestra Señora de Candelaria, 38010 Santa Cruz de Tenerife; \\ ${ }^{2}$ Nefrologia Infantil, Hospital General Univesitario Gregorio Marañón, 28007 Madrid, Spain
}

Received June 18, 2020; Accepted August 20, 2020

DOI: $10.3892 /$ br.2020.1356

\begin{abstract}
Nail-Patella syndrome (NPS) is an inherited disease characterized by nail and skeletal anomalies, nephropathy and glaucoma. The diagnosis of NPS is based on clinical findings, including hypoplastic or absent patella, dystrophic nails, dysplasia of the elbows and iliac horns. However, the main determinant of NPS prognosis is nephropathy, which may range from asymptomatic proteinuria to end-stage renal disease. NPS is caused by heterozygous loss-of-function mutations in the $L M X 1 B$ gene, which encodes the LIM homeodomain transcription factor LMX1B. LMX1B serves an essential role in the physiological development of dorsal-ventral limb structures, morphogenesis and function of podocytes, as well as in development of the anterior segments of the eyes, and in certain types of neurons. The present study aimed to identify the disease-causing mutation in a 2-year old girl with nephrotic syndrome that evolved rapidly to end-stage renal disease. The patient showed classical symptoms of NPS including dystrophic nails and an absence of the patellae. DNA sequence analysis identified a novel missense variant in exon 4 of $L M X 1 B$ (c.709T>C, p.S237P); this substitution affected a conserved serine residue in the homeodomain of LMX1B and was predicted to be pathogenic. In silico modeling of the homeodomain revealed that the p.S237P mutation converted the A236-S237-F238 segment of $\alpha$-helix 1 into a strand. It was hypothesized that this mutation affected binding of the transcription factor to its target DNA, thus abrogating transcription activation, which would explain the phenotype that manifested in the patient.
\end{abstract}

Correspondence to: Dr Felix Claverie-Martin, Unidad de Investigación, Hospital Universitario Nuestra Señora de Candelaria, Carretera del Rosario 145, 38010 Santa Cruz de Tenerife, Spain E-mail: fclamar@gobiernodecanarias.org

Key words: LMX1B, nail-patella syndrome, homeodomain, missense mutation, nephropathy, end-stage renal disease

\section{Introduction}

Nail-Patella syndrome (NPS) or hereditary onycho-osteodysplasia (OMIM entry no. 161200) is a rare autosomal dominant disease that affects multiple organs including the limbs, skeleton, kidneys and eyes (1). The classical clinical manifestations consist of nail dysplasia, hypoplasia or absence of the patella, elbow dysplasia and iliac horns (1). Renal involvement occurs in 30-50\% of patients with NPS and is the primary determinant of prognosis $(2,3)$. Renal manifestations typically include proteinuria with or without hematuria, and nephrotic syndrome (5-10\% of cases), which progresses to end-stage renal disease (ESRD) over varying periods of time (3). Additional relevant characteristics observed in patients with NPS include open angle glaucoma and ocular hypertension, which usually develop during adulthood and occur in $\sim 10$ and $7 \%$ of patients, respectively $(2,4)$. The gene associated with NPS, $L M X 1 B$, maps on to chromosome 9q33.3 and contains 8 exons that encode a LIM-homeodomain transcription factor called LMXB1 (5-7). This protein belongs to the LIM-homeodomain protein family, and serves an essential role in the normal development of dorsal-ventral limb structures, morphogenesis, and in the functions of podocytes and the glomerular basal membrane, anterior segment of the eye, and some types of neurons $(1,5)$.

LMX1B contains two cysteine-rich zinc-binding motifs (LIM-A and LIM-B domains) at the N-terminus, which are all involved in protein-protein interactions; a homeodomain in the middle responsible for the interaction with specific DNA sequences in target genes, and a C-terminal glutamine-rich domain of unknown function (1,7). Most loss-of-function mutations of LMX1B are clustered in the LIM domains and the homeodomain, encoded by exons 2-3 and 4-6, respectively (8-11). Several studies have reported entire deletions of the $L M X 1 B$ gene in patients with NPS, supporting haploinsufficiency as the major pathogenic mechanism underlying the disease $(8,9,12,13)$. However, a clear genotype-phenotype correlation among NPS patients is still lacking (8). Even patients within the same family present a varying range of symptoms and symptom severity, emphasizing the potential contribution of modifier genes in the manifestation of the phenotype (11). 
In the present case report, an unusual case of a 2-year old girl from Syria who presented with nephrotic syndrome that evolved rapidly to ESRD is described. She exhibited classical NPS symptoms including dystrophic nails and absence of patellae. Using DNA sequencing analysis, a novel heterozygous missense mutation in $L M X 1 B$ (c.709T >C, p.S237P), which affected the homeodomain of the LMX1B protein was identified, which was predicted to be pathogenic.

\section{Case report}

Subject. The patient was a 2-year-old girl from Syria. She was admitted to the Hospital General Univesitario Gregorio Marañón (Madrid, Spain) for conventional hemodialysis. Genetic studies were performed after obtaining written consent from the parents. The Ethics Committee of Hospital Universitario Nuestra Señora de Candelaria (Santa Cruz de Tenerife, Spain) approved the present study. The clinical study included radiological examination, biopsy and an ophthalmological exam.

Clinical manifestations. The patient presented with nephrotic syndrome at the age of 2 years, which did not respond to steroid treatment. The family history was unremarkable and the patient has one healthy sister 2 years older than her. She was born after a normal pregnancy; her weight and height were $3.8 \mathrm{~kg}$ and $52 \mathrm{~cm}$, respectively. She received breastfeeding and showed adequate psychomotor development. A renal biopsy performed when she was 2 years and 2 months old revealed membranoproliferative glomerulonephritis type I. She was diagnosed with steroid-resistant nephrotic syndrome in the context of NPS. Therefore, corticosteroid treatment was discontinued gradually. After the gradual progression of the disease a second biopsy showed evolution to glomerulosclerosis and glomerular fibrosis. The patient progressed rapidly to ESRD 8 months after the first signs of renal impairment. She was placed under conventional hemodialysis and 2 months later under peritoneal dialysis until she received a renal transplant when she was 4 years old. Recently, the patient reentered hemodialysis due to chronic graft rejection and was transplanted again. The orthopedic signs of NPS exhibited by the patient were dystrophic nails and absence of patellae observed by X-ray examination (Fig. 1). The patient was noted to have coxa valga on X-ray (data not shown), although this is not a characteristic of NPS. Ophthalmological examination showed no ocular defects.

\section{Materials and methods}

DNA amplification and Sanger sequencing. Genomic DNA was extracted from peripheral blood samples using the GenElute Blood Genomic DNA kit according to the manufacturer's protocol (Sigma-Aldrich; Merck KGaA). DNA quality and quantity were assessed using a NanoDrop Lite spectrophotometer (Thermo Fisher Scientific, Inc.). For analysis of mutations in the $L M X 1 B$ gene, the 8 coding exons and their intronic flanking sequences were amplified by PCR as previously described (14-15). PCR products were analyzed by agarose gel (1.5\%) electrophoresis, and the fragments were purified using QIAquick PCR purification kit (Qiagen, Inc.). DNA sequencing of purified products was performed by Macrogen Inc. Variants were identified by comparison to the respective $L M X 1 B$ reference sequences

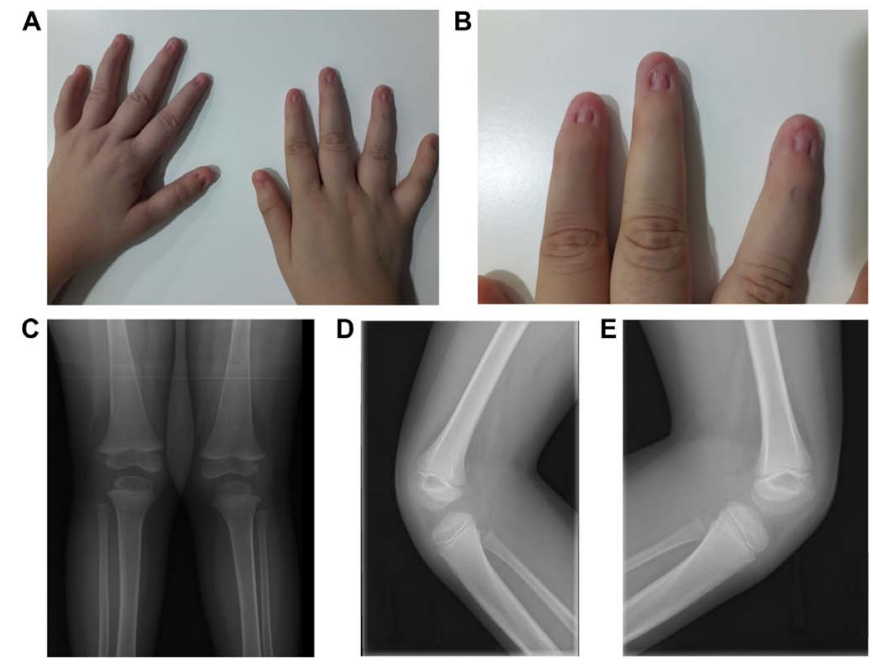

Figure 1. Nail abnormalities and orthopedic manifestations in the patient with Nail-Patella syndrome. (A and B) Dystrophic nails were observed on both hands. (C-E) X-ray examination showing the absence of the patella.

(GeneBank accession no. NG_17039.1), and confirmed by sequencing additional independent amplification products.

Bioinformatics analysis. Several databases, including gnomAD (gnomad.broadinstitute.org/), 1000 Genomes Project (1000genomes.org/), Human Gene Mutation Database (HGMD, hgmd.cf.ac.uk/ac/index.php) and ClinVar (ncbi.nlm. nih.gov/clinvar/), were explored to determine whether the mutation detected in the present study had been reported previously. Online bioinformatics tools PolyPhen-2 (genetics.bwh. harvard.edu/pph2), Align GVGD (agvgd.iarc.fr), MutPred2 (mutpred.mutdb.org), Mutation Taster (mutationtaster.org) and VarSome (varsome.com), a new search engine for human genomic variation (16), were used to predict the pathogenicity of the identified mutation. The protein sequence of human LMX1B (isoform 2 containing 402 amino acids) was obtained from the NCBI database (accession no. NP_001167618.1). The protein structure homology-modelling server SWISS-MODEL was used to model the 3D structure of the wild-type and mutant LMX1B homeodomains using LIM/Homeobox protein Lhx4 as a template.

\section{Results}

Based on the orthopedic manifestations and the renal course of the patient, mutational analysis of the $L M X 1 B$ gene was performed. The results of the DNA sequencing analysis showed a novel heterozygous variant in exon 4 of $L M X 1 B$, c.709T $>C$ (g.78849T>C, genomic location Chr9; 129455570>C GRCh37; Fig. 2A). This nucleotide substitution was predicted change a serine residue to proline at position 237 (p.S237P) in the homeodomain of the LMX1B protein (Ensembl transcript ID, ENST00000355497). Since blood samples from the patient's parents were not available, it was not possible to determine if the mutation was inherited from one of parents or if it originated de novo. Variant c.709T>C, p.S237P was absent from several databases, including gnomAD, 1000 Genomes Project, HGMD and ClinVar. Homology analysis showed that serine 237 of LMX1B is conserved amongst different species (Fig. 2B). Analysis of 
Table I. Pathogenicity prediction of the p.S237P mutation in LMX1B using various bioinformatics tools.

\begin{tabular}{lccccc}
\hline Outcomes & PolyPhen-2 $^{\mathrm{a}}$ & Align GVGD $^{\mathrm{b}}$ & MutPred2 $^{\mathrm{c}}$ & Mutation Taster $^{\mathrm{d}}$ & VarSome $^{\mathrm{e}}$ \\
\hline $\begin{array}{l}\text { Score } \\
\text { Prediction }\end{array}$ & 1.0 & Class 65 & 0.886 & 74 & - \\
Probably damaging & Most likely damaging & Likely pathogenic & Disease causing & Likely pathogenic
\end{tabular}

aPolyPhen-2 score range, 0.0-1.0. Variants with scores in the range $0.85-1.0$ are more confidently predicted to be damaging. ${ }^{\mathrm{b}} \mathrm{Align}-\mathrm{GVGD}$ classifies variants in seven risk grades (Class $0,15,25,35,45,55$ or 65 ) with Class 65 most likely to interfere with function and Class 0 least likely. ${ }^{\mathrm{c}}$ MutPred 2 prediction scores range from 0.0-1.0; a higher score indicated a greater likelihood of pathogenicity. ${ }^{\mathrm{d}}$ MutationTaster scores range from 0.0-215. The scores are calculated based on the Grantham Matrix for amino acid substitutions, and reflects the physicochemical difference between the original and the mutated amino acid. ${ }^{\mathrm{e}}$ The mutation met 2 moderate (PM2 and PM3) and 3 supporting (PP2, PP3 and PP5) criteria.
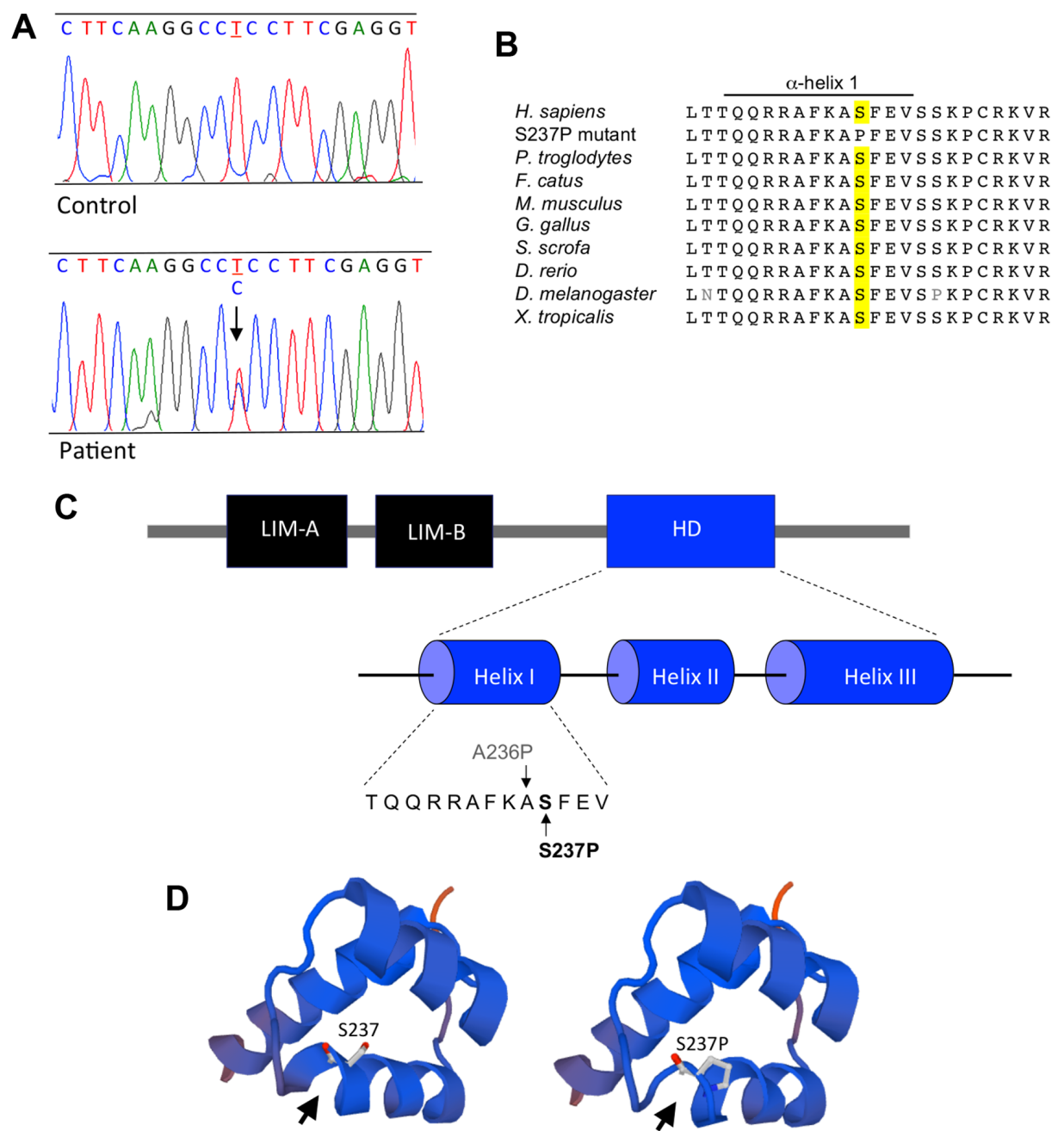

Figure 2. Identification of a novel $L M X 1 B$ mutation, c.709T $>$ C, p.S237P, and prediction of the effect of the mutation on the 3D structure of the protein (A) Electropherograms showing the c.709T $>$ C, p.S237P heterozygous change in $L M X 1 B$ exon 4 of the patient (indicated by an arrow) and control sequence. (B) Multiple sequence alignment of the LMX1B homeodomain from different species of vertebrates and a predicted protein from D. melanogaster showing the evolutionary conservation of the serine residue at position 237 (highlighted in yellow). (C) Diagram of the LMX1B protein showing the functional domains (LIM domains and HD) and the location of p.S237P. The HD (amino acid residues 219-278) is composed of $\alpha$-helices 1,2 and 3. The position of the novel mutation p.S237P (in bold) and the previously reported mutation p.A236P, also located in helix 1, are indicated. (D) Models of the wild-type and mutant S237P homeodomains of LMX1B were constructed with SWISS-MODEL using LIM/Homeobox protein Lhx4 as the template. The helical structure of the segment A236-S237-F238 was lost and converted into a strand (arrow heads). HD, homeodomain.

p.S237P with five different bioinformatics tools predicted that this variant was pathogenic (Table I). VarSome classified this substitution as likely pathogenic as it met 2 moderate (PM2 and PM3) and 3 supporting (PP2, PP3 and PP5) pathogenicity criteria established by the American College of Medical Genetics and
Genomics (17). The novel $L M X 1 B$ mutation described here was submitted to ClinVar (accession no. VCV000694525; variant, NM_002316.4; ncbi.nlm.nih.gov/clinvar/variation/694525/). As shown in Fig. 2C, the missense mutation p.S237P is located in $\alpha$-helix 1 of the LMX1B homeodomain. To evaluate the structural 
impact of this mutation, 3D models of the wild-type and mutant homeodomains were constructed using SWISS-MODEL. The results suggested that the substitution of serine for proline at position 237 transformed the segment A236-S237-F238 of the $\alpha$-helix 1 in the homeodomain into a strand (Fig. 2D).

\section{Discussion}

NPS is an autosomal dominant disorder characterized by dysplastic nails, a hypoplastic or absent patella, and elbow dysplasia $(1,2)$. In some cases, patients may also exhibit differing levels of proteinuria that can progress toESRD, and less frequently open-angle glaucoma. The skeletal disorders can be detected at birth, but in some cases they are less significant, making the diagnosis problematic $(1,3)$. In the present case report, NPS was challenging to diagnose immediately as nephrotic syndrome developed before obvious orthopedic symptoms, and there was no family history of NPS. This case was quite uncommon as the patient presented persistent nephrotic syndrome very early, at the age of 2 years, and developed rapidly to ESRD. The majority of patients with NPS exhibit a slow gradual loss of renal function, and only a small number of cases, where a rapid progression to ESRD at a young age, have been reported $(15,18)$. The factors responsible for progression to ESRD in NPS are unknown, but it is possible that potential modifying genes may contribute to it. The nephropathy in the patient reported on in the present case report was treated with corticosteroids and diuretics, and the corticoresistance supported the association of the nephrotic syndrome with NPS. Moreover, renal biopsy of the patient revealed a characteristic irregular thickening of the glomerular basement membrane with progression to fibrosis and glomerulosclerosis observed in other patients with NPS (3). Additionally, the nail and skeletal phenotype of the patient was consistent with previously published data of NPS (2).

NPS is caused by loss-of-function mutations in the $L M X 1 B$ gene, which is expressed in several different tissues implicated in the clinical phenotype (5-7). In the kidney, LMX1B is expressed in podocytes and regulates the expression of several genes required for correct podocyte differentiation and function (1). Studies using inducible podocyte specific LmxIb knockout mice have established the importance of LMX1B in fully differentiated podocytes, and suggest that this transcription factor is essential for the maintenance of a properly structured actin cytoskeleton (19). Additionally, LMX1B has been shown to bind enhancer sequences (FLAT elements) in the promoter regions of $A B R A$ and $A R L 4 C$, two genes encoding actin cytoskeleton-associated proteins (19). These studies concluded that podocyte pathogenesis in NPS likely results from a dysregulation of the actin cytoskeleton.

During embryogenesis, LMX1B expression is high in the dorsal mesenchyme of developing limbs and is involved in dorso-ventral patterning (20). Following the formation of the dorsal-ventral axis, LMX1B activates the expression of several dorsalizing genes, represses the expression of ventralizing genes, and regulates the development of dorsal distal limb structures, such as the nails and patellas (20). Therefore, the skeletal phenotype of patients with NPS is the result of a defect in dorsoventral patterning caused by $L M X 1 B$ mutations.

LMX1B is also expressed during ocular development in the anterior portions of the murine eye $(21) ; \sim 10 \%$ of NPS patients develop open-angle glaucoma and an additional $7 \%$ exhibit ocular hypertension $(2,8)$. These ocular symptoms usually develop during adulthood. The patient reported in the present case report is now 14 years old and she has not yet developed glaucoma. She undergoes ophthalmological reviews every 6 months. The pathogenesis of glaucoma in NPS is still unknown, but homozygous $\operatorname{lm} x 1 b$ knockout mice display iris and ciliary body hypoplasia and corneal collagen fibril defects, suggesting a role for LMX1B in collagen regulation during development (21).

Several of the mutations identified in patients with NPS are localized in the homeodomain of $\operatorname{LMX} 1 B(8,9,11)$. This highly conserved DNA-binding motif forms part of several transcription factors present in numerous organisms, from yeast to humans (22). These transcription factors serve essential roles during development and adult homeostasis, and mutations in their genes are known to cause human diseases. These genes include $H O X A 13$ and $H O X D 13$, which are associated with skeletal defects in distal extremities; $L H X 3$ and POU1F1, which are associated with pituitary hormone deficiency; and PAX6 which is associated with eye-related disorders (22). The homeodomain is comprised of $\sim 60$ amino acids and includes three $\alpha$-helices, of which helices 2 and 3 adopt a helix-turn-helix motif, and a flexible N-terminal arm. Helix 3 and the N-terminal arm interact directly with the major groove and the adjacent minor groove of target DNAs, respectively, whereas helices 1 and 2 maintain the tertiary structure important in the helix-turn-helix configuration. Mutational analysis of $L M X 1 B$ in the present case report identified a novel heterozygous variant, c.709T $>C$, p.S237P, that explained the phenotype observed. This variant, which was not found in any of the reference databases, resulted in a change in a conserved serine residue to a helix-disrupting proline residue, and was predicted to be pathogenic by several different bioinformatics tools. The evolutionary conservation of serine 237 indicates that this residue is essential for the structure and function of the LMX1B homeodomain. Although mutational analysis of the patient's parents was not possible, it is hypothesized that p.S237P is a de novo mutation as there was no history of nephropathy, skeletal defects or nail dystrophy in either parent, sister, grandparents or uncles. The identified mutation is located next to the amino acid residue affected by recurrent mutation c.706G $>$ C, p.A236P in $\alpha$-helix 1 of the homeodomain and appears as p.A213P in (6). p.A236P has been shown to abolish both DNA binding of the LMX1B homeodomain to the FLAT element of the insulin promoter and transactivation of a reporter gene $(6,12)$. Knoers et al $(23)$ indicated that p.A236P disrupted the 3D structure of the homeodomain and, therefore, disrupted DNA binding. Similarly, p.S237P also introduces a proline residue that may destabilize the helix conformation and affect the overall tertiary structure of the homeodomain. Analysis of the predicted structural model of the mutant homeodomain in the present study indicated that p.S237P indeed disturbed the carboxy-terminal segment of $\alpha$-helix 1. Thus, together, these results suggests that p.S237P destabilizes $\alpha$-helix 1, abolishing DNA binding and the subsequent transcriptional activity of LMX1B. This hypothesis requires confirmation using functional studies.

In conclusion, a novel missense mutation (c.709T $>C$, p.S237P) in the $L M X 1 B$ gene of a child presenting with nephrotic syndrome and rapid progression to ESRD was identified. Based on in silico structural analysis of this mutation, 
the $\alpha$-helix 1 was altered, which abrogated binding of the LMX1B homeodomain to the target DNA. This report further expands on the mutational spectrum of $L M X 1 B$, and provides insight into the molecular mechanisms of NPS pathology.

\section{Acknowledgements}

Not applicable.

\section{Funding}

This work was supported by integrated in the Plan Nacional de I+D+I 2013-2016 (grant no. PI17/00153), and co-financed by the ISCIII-Subdirección General de Evaluación y Fomento de la Investigación and the European Regional Development Fund 'Another way to build Europe'.

\section{Availability of data and materials}

The datasets used and/or analyzed during the present study are available from the corresponding author on reasonable request. The novel $L M X 1 B$ mutation described in the present has been submitted to ClinVar (accession no. VCV000694525; variant, NM_002316.4.

\section{Authors' contributions}

SC and APR performed PCR amplifications and analyzed the DNA sequences. SC, APR and FCM carried out the bioinformatics analysis. OAB collected the patient's data and followed up the case. SC and FCM wrote the manuscript. All authors read and approved the final manuscript.

\section{Ethics approval and consent to participate}

This study was approved by the Ethics Committee of Hospital Universitario Nuestra Señora de Candelaria (Santa Cruz de Tenerife, Spain) (approval no. C.P. MO PI17/00153-C.I. PI56-17). Written informed consent for participation of the child in the genetic study was obtained from her parents.

\section{Patient consent for publication}

Written informed consent for publication of this study was obtained from the parents of the child.

\section{Competing interests}

The authors declare that they have no competing interests.

\section{References}

1. Witzgall R: Nail-patella syndrome. Pflugers Arch 469: 927-936, 2017.

2. McIntosh I, Dunston JA, Liu L, Hoover-Fong JE and Sweeney E: Nail patella syndrome revisited: 50 years after linkage. Ann Hum Genet 69: 349-363, 2005

3. Lemley KV: Kidney disease in nail-patella syndrome. Pediatr Nephrol 24: 2345-2354, 2009.

4. Lichter PR, Richards JE, Downs CA, Stringham HM, Boehnke M and Farley FA: Cosegregation of open-angle glaucoma and the nail-patella syndrome. Am J Ophthalmol 124: 506-515, 1997.
5. Dreyer SD, Zhou G, Baldini A, Winterpacht A, Zabel B, Cole W, Johnson RL and Lee B: Mutations in LMX1B cause abnormal skeletal patterning and renal dysplasia in nail patella syndrome. Nat Genet 19: 47-50, 1998.

6. McIntosh I, Dreyer SD, Clough MV, Dunston JA, Eyaid W, Roig CM, Montgomery T, Ala-Mello S, Kaitila I, Winterpacht A, et al: Mutation analysis of LMX1B gene in nail-patella syndrome patients. Am J Hum Genet 63: 1651-1658, 1998.

7. Vollrath D, Jaramillo-Babb VL, Clough MV, McIntosh I, Scott KM, Lichter PR and Richards JE: Loss-of-function mutations in the LIM-homeodomain gene, LMX1B, in nail-patella syndrome. Hum Mol Genet 7: 1091-1098, 1998

8. Bongers EM, Huysmans FT, Levtchenko E, de Rooy JW, Blickman JG, Admiraal RJ, Huygen PL, Cruysberg JR, Toolens PA, Prins JB, et al: Genotype-phenotype studies in nail-patella syndrome show that LMX1B mutation location is involved in the risk of developing nephropathy. Eur J Hum Genet 13: 935-946, 2005.

9. Marini M, Bocciardi R, Gimelli S, Di Duca M, Divizia MT, Baban A, Gaspar H, Mammi I, Garavelli L, Cerone R, et al: A spectrum of LMX1B mutations in Nail-Patella syndrome: New point mutations, deletion, and evidence of mosaicism in unaffected parents. Genet Med 12: 431-439, 2010.

10. Hamlington JD, Jones $C$ and McIntosh I: Twenty-two novel LMX1B mutations identified in nail patella syndrome (NPS) patients. Hum Mutat 18: 458, 2001.

11. Ghoumid J, Petit F, Holder-Espinasse M, Jourdain AS, Guerra J, Dieux-Coeslier A, Figeac M, Porchet N, Manouvrier-Hanu S and Escande F: Nail-Patella Syndrome: Clinical and molecular data in 55 families raising the hypothesis of a genetic heterogeneity. Eur J Hum Genet 24: 44-50, 2016.

12. Dreyer SD, Morello R, German MS, Zabel B, Winterpacht A, Lunstrum GP, Horton WA, Oberg KC and Lee B: LMX1B transactivation and expression in nail-patella syndrome. Hum Mol Genet 9: 1067-1074, 2000

13. Sato U, Kitanaka S, Sekine T, Takahashi S, Ashida A and Igarashi T: Functional characterization of LMX1B mutations associated with nail-patella syndrome. Pediatr Res 57: 783-788, 2005.

14. Claverie-Martin F, Trindade A, Garcia-Gonzalez NC and Callejon AC: Novel missense mutation affecting the LIM-A domain of LMX1B in a family with Nail-Patella syndrome. Intractable Rare Dis Res 8: 14-19, 2019.

15. Lee BH, Cho TJ, Choi HJ, Kang HK, Lim IS, Park YH, Ha IS, Choi Y and Cheong HI: Clinico-genetic study of nail-patella syndrome. J Korean Med Sci 24 (Suppl 1): S82-S86, 2009.

16. Kopanos C, Tsiolkas V, Kouris A, Chapple CE, Albarca Aguilera M, Meyer R and Massouras A: VarSome: The human genomic variant search engine. Bioinformatics 35: 1978-1980, 2019.

17. Richards S, Aziz N, Bale S, Bick D, Das S, Gastier-Foster J, Grody WW, Hegde M, Lyon E, Spector E, et al: Standards and guidelines for the interpretation of sequence variants: A joint consensus recommendation of the American college of medical genetics and genomics and the association for molecular pathology. Genet Med 17: 405-424, 2015.

18. Bongers EM, Gubler MC and Knoers NV: Nail-patella syndrome. Overview on clinical and molecular findings. Pediatr Nephrol 17: 703-712, 2002.

19. Burghardt T, Kastner J, Suleiman H, Rivera-Milla E, Stepanova N Lottaz C, Kubitza M, Böger CA, Schmidt S, Gorski M, et al: LMX1B is essential for the maintenance of differentiated podocytes in adult kidneys. J Am Soc Nephrol 24: 1830-1848, 2013.

20. Chen H, Lun Y, Ovchinnikov D, Kokubo H, Oberg KC, Pepicelli CV, Gan L, Lee B and Johnson RL: Limb and kidney defects in Lmx1b mutant mice suggest an involvement of LMX1B in human nail patella syndrome. Nat Genet 19: 51-55, 1998.

21. Pressman CL, Chen H and Johnson RL: LMX1B, a LIM homeodomain class transcription factor, is necessary for normal development of multiple tissues in the anterior segment of the murine eye. Genesis 26: 15-25, 2000.

22. Chi YI: Homeodomain revisited: A lesson from disease-causing mutations. Hum Genet 116: 433-444, 2005

23. Knoers NV, Bongers EM, van Beersum SE, Lommen EJ, van Bokhoven $\mathrm{H}$ and Hol FA: Nail-patella syndrome: Identification of mutations in the LMX1B gene in Dutch families. J Am Soc Nephrol 11: 1762-1766, 2000. International (CC BY-NC-ND 4.0) License. 